\title{
Portadores asintomáticos de hidatidosis: epidemiología, diagnóstico y tratamiento
}

\author{
Edmundo Larrieu, ${ }^{1}$ Bernardo Frider, ${ }^{2}$ Mario del Carpio, ${ }^{3}$ \\ Juan C. Salvitti, ${ }^{4}$ Carlos Mercapide, ${ }^{5}$ Rubén Pereyra, ${ }^{5}$ \\ María Costa, ${ }^{3}$ Martín Odriozola, ${ }^{4}$ Alicia Pérez, ${ }^{6}$ \\ Gustavo Cantoni ${ }^{6}$ y José Sustercic ${ }^{7}$
}

RESUMEN Hasta hace poco tiempo, la hidatidosis se consideraba un estado patológico de resolución exclusivamente quirúrgica. Sin embargo, en los últimos años se ha avanzado en los campos de la epidemiología, el diagnóstico y el tratamiento de la enfermedad y la nueva información aportada sobre la historia natural de la hidatidosis ha permitido definir nuevos criterios de atención. Ahora se sabe que hasta $67 \%$ de los portadores no sintomáticos de quistes hepáticos mantienen esa condición durante toda la vida. Esta situación genera resultados especiales en el inmunodiagnóstico. Así, la inmunoadsorción enzimática (ELISA) rinde una sensibilidad de 63\% y una especificidad de $97 \%$ en portadores asintomáticos, mientras que la doble difusión cinco (DD5) tiene una sensibilidad de solo $31 \%$ en esos portadores. Por otra parte, los estudios por imágenes basados en la ecografía se han transformado en el método de elección para detectar a los portadores no sintomáticos. Son de 49 a 73\% más sensibles que la serología e incluso pueden utilizarse como parte del sistema de vigilancia epidemiológica y del monitoreo de programas de control. También se han modernizado los esquemas de intervención. El tratamiento quimioterápico de portadores asintomáticos con albendazol produce hasta $69 \%$ de respuestas favorables, mientras que los tratamientos quirúrgicos mínimamente invasores como la punción-aspiracióninyección-reaspiración (PAIR) producen una reducción del volumen medio del quiste de hasta $66 \%$. Estos factores han permitido instaurar un protocolo de tratamiento para portadores asintomáticos en los servicios hospitalarios de la Provincia de Río Negro, Argentina. Este se basa en el seguimiento de los quistes pequeños (tipo Ia de la escala de Gharbi modificada), tratamiento inicial con albendazol y con PAIR si no hay respuesta, en los quistes de mayor tamaño o complejidad (tipos Ib, II y III), y seguimiento de los quistes no vitales o muertos (tipos IV y $V)$.

1 Consejo Provincial de Salud Pública, Provincia de Río Negro, Argentina. Toda la correspondencia debe dirigirse a Edmundo Larrieu, a la siguiente dirección postal: Laprida 240 (8599) Viedma, Argentina. Correo electrónico: msrione@anmat. gov.ar

2 Hospital Argerich, Buenos Aires, Argentina.

3 Hospital Cortizo, Ing. Jacobacci, Río Negro, Argentina.

4 Hospital Carrillo, Bariloche, Río Negro, Argentina.

5 Hospital Zatti, Viedma, Río Negro, Argentina.

6 Zona sanitaria IV, Bariloche, Río Negro, Argentina.

Hospital López Lima, General Roca, Río Negro, Argentina.
La hidatidosis es una zoonosis parasitaria de alta endemicidad en algunos países de América del Sur, sobre todo en la Argentina, Chile, Uruguay y Brasil. Producida por la forma larval de Echinococcus granulosus, se localiza principalmente en el hígado y los pulmones. Tradicionalmente, la hidatidosis se ha considerado un estado patológico esencialmente de resolución quirúrgica. Sin embargo, en los últi- mos años, los avances en el campo de la epidemiología han aportado nueva información sobre la historia natural de la enfermedad, tal como la existencia de un elevado número de portadores que permanecen asintomáticos durante toda la vida. En respuesta a los conocimientos actuales han surgido nuevos criterios de diagnóstico y tratamiento. Seguidamente se presenta una revisión de las experiencias desarrolla- 
das por los servicios de salud de la Provincia de Río Negro, Argentina, en el diagnóstico y tratamiento de portadores de quistes hidatídicos sin síntomas clínicos de la enfermedad.

\section{Epidemiología de la hidatidosis}

El cestodo Echinococcus granulosus en su forma adulta vive en el intestino del perro y otros cánidos y elimina huevos periódicamente con la materia fecal. Estos huevos pueden ser ingeridos accidentalmente por los huéspedes intermediarios, entre ellos el hombre. Los huevos eclosionan, liberando el embrión hexacanto en el intestino delgado. Este pasa a través de las vellosidades intestinales a la circulación venosa hasta alojarse en el tejido hepático donde forma una hidátide. A las 72 horas después de haberse ingerido el huevo, la larva alcanza un tamaño de 20 micrones, mientras que a los 7 días puede identificarse una formación esférica y claramente hidatídica (1).

En caso de traspasar el filtro hepático, el embrión continúa por el sistema circulatorio hasta los pulmones. Posteriormente puede continuar su migración y alcanzar diversas localizaciones como los riñones, el cerebro o el tejido óseo o muscular (1).

El crecimiento del parásito depende del potencial evolutivo del embrión hexacanto, las características del tejido circundante y la resistencia del huésped. El pulmón, por su elasticidad, ofrece poca resistencia al desarrollo del embrión. Este logra un aumento de tamaño relativamente rápido, lo que lleva a la aparición de síntomas clínicos en un gran porcentaje de los casos. Por contraste, en el hígado la resistencia del tejido circundante es fuerte, lo que determina que el crecimiento del embrión sea lento o incluso nulo durante muchos años. Por esta razón, una alta proporción de portadores permanecen sin síntomas clínicos durante toda la vida.

En los servicios hospitalarios que reciben pacientes con síntomas clínicos, las localizaciones de hígado y pulmón que se observan se diferencian a razón de 2:1 a 3:1. Las localizaciones en otros órganos no pasan de $10 \%$ de los casos detectados. Estos coeficientes expresan la importancia del filtro hepático para retener las larvas y determinar la localización del quiste.

Los casos de hidatidosis detectados durante autopsias muestran una relación de 4:1 entre las localizaciones hepática y pulmonar. No obstante, de 63,3 a $84,5 \%$ de los casos hallados no guardan relación alguna con la causa de muerte (2). Esta cifra apoya el criterio de que el filtro hepático retiene las larvas y es el factor primordial que determina la localización del parásito e indica que un porcentaje importante de los quistes hepáticos no llegan a producir enfermedad en el huésped, sino que mantienen un estado de equilibrio entre parásito y huésped durante toda la vida (3).

La ejecución consecutiva de radiografía del tórax y ecografía abdominal en muestras representativas de población suele eliminar los sesgos de las notificaciones hospitalarias y de la poca representatividad de las autopsias en la población general y reportar cifras más reales de la relación entre las localizaciones hepática y pulmonar. Por ejemplo, en las provincias de Río Negro y Neuquén en la Argentina, el tamizado de 647 personas permitió calcular una relación entre la localización hepática y pulmonar de 5:1. En el Uruguay se han registrado tasas de prevalencia de hidatidosis hepática de $1,6 \%$ y de hidatidosis pulmonar de $0,2 \%$ en una muestra de 9481 personas (4-6). La detección preliminar de casos con la técnica de doble difusión cinco (DD5) en una población sin síntomas clínicos permitió confirmar por imaginología 5,4 quistes hidatídicos hepáticos por cada quiste pulmonar (2).

En un estudio llevado a cabo en la Provincia de Río Negro, se siguieron durante 14 años mediante ultrasonografía los portadores asintomáticos de quistes hidatídicos detectados en tamizados efectuados de grupos de población general. De los casos, 67\% mantuvieron su condición asintomática y los quistes crecieron en promedio $3 \mathrm{~cm} \mathrm{o}$ menos. Sin embargo, 33\% de los casos necesitaron intervención quirúrgica debido a la aparición de síntomas clínicos producidos por el crecimiento notable de los quistes (7). Esta información refuerza la teoría de que existe un estado de equilibrio entre parásito y huésped en un porcentaje importante de los portadores de quistes hidatídicos de localización hepática.

\section{Respuesta inmunitaria e inmunodiagnóstico}

En el hombre, el líquido hidatídico es el principal factor responsable de la estimulación antigénica. Por el contrario, la capa laminar, acelular y no degradable no estimula el sistema inmunitario del huésped, aunque se comporta como un filtro que permite el paso de macromoléculas. Por su parte, el tegumento de la capa germinativa actúa como barrera que impide el contacto de los antígenos del líquido hidatídico con las células inmunocompetentes del huésped. Es necesario que en la capa germinativa ocurran alteraciones como microfisuras, complicaciones o roturas que modifiquen sus condiciones de permeabilidad para que la estimulación antigénica se produzca y pueda ser detectada por métodos inmunológicos (8).

Por las características ya mencionadas, los quistes de localización pulmonar tienen menos posibilidades de adquirir microfisuras en la pared. En cuanto al diagnóstico inmunológico, ello implica que los quistes pulmonares den resultados preponderantemente negativos en las pruebas serológicas usuales. En los quistes hepáticos hay más posibilidades de microfisuras y complicaciones y, así también, de resultados serológicos positivos. Por su parte, los quistes pequeños y hialinos de cualquier localización tienen una baja posibilidad de salida de inmunógenos y por lo general dan resultados negativos.

Entre pacientes sintomáticos con hidatidosis confirmada por cirugía, 80\% de los casos han dado resultados serológicos positivos en las pruebas de hemaglutinación indirecta (HAI), de 82 a $88 \%$ resultaron positivos en las de DD5, $82 \%$ en la inmunoelectroforesis, de 88 a $96 \%$ en la inmunoadsorción enzimática (ELISA) y 92\% en la inmunoelectrotransferencia. La especificidad de 
estos métodos varía desde $95 \%$ en la HAI hasta $100 \%$ en la DD5 (9-14).

En portadores sin síntomas clínicos, la información es muy limitada, aunque la posibilidad de detectar una respuesta serológica positiva es mucho menor. Por ejemplo, en la provincia de Río Negro, la sensibilidad y especificidad de las pruebas DD5 y ELISA se han corroborado por medio de estudios completos de imaginología (radiografía, ecografía y tomografía) como pruebas de referencia. Los resultados fueron 63 y 97,05\%, respectivamente, para el ELISA a títulos iguales a $8 \mathrm{DE}$ o más, y 31 y 100\%, respectivamente, para la DD5 (15) (cuadro 1).

\section{Diagnóstico de portadores asintomáticos mediante ecografía}

La utilidad y la validez de la ecografía en el diagnóstico de la hidatidosis humana han sido ampliamente verificadas tanto en pacientes sintomáticos como en portadores asintomáticos (16-22). Desde el punto de vista de las imágenes ecográficas del quiste hidatídico, se consideran patognomónicas las siguientes características: a) vesículas aisladas; b) vesículas hijas múltiples; c) observación del "nevado" de la arenilla hidatídica al mover bruscamente al paciente 180 grados (20); d) aparición de membranas desprendidas, y e) pared del quiste hidatídico de mayor espesor que en los quistes serosos simples o en la enfermedad poliquística. En condiciones operativas de campo para la detección de portadores de quistes hidatídicos de localización hepática, la sensibilidad y especificidad de la ecografía se han estimado en $100 \%$ y 96 a $97 \%$, respectivamente $(18,23)$.

Gharbi (17) ha elaborado un esquema de clasificación basado en cinco tipos principales de imágenes ecográficas de quistes hidatídicos. Estas imágenes pueden asimilarse al estado evolutivo del quiste, lo cual resulta especialmente útil para la selección posterior del tratamiento. Este esquema, ajustado, se aplica en los servicios de salud de la provincia de Río Negro:
- Tipo Ia: quistes hialinos de menos de $3 \mathrm{~cm}$ de diámetro, con o sin signo del nevado y menos de $20 \mathrm{~cm}^{3}$ de contenido líquido parecido al agua. Constituyen quistes vitales.

- Tipo Ib: quistes hialinos de más de 3 $\mathrm{cm}$ de diámetro, con o sin signo del nevado, membrana visible y contenido líquido parecido al agua. Constituyen quistes vitales.

- Tipo II: quistes de cualquier tamaño, con desprendimiento de la membrana y líquido como agua. Constituyen quistes vitales, con indicaciones de sufrimiento.

- Tipo III: quistes de diámetro variable, líquido como pus y vesículas múltiples en su interior. Constituyen quistes cuya vitalidad puede estar disminuyendo, pero son muy agresivos y tienen mayores posibilidades de complicarse.

- Tipo IV: quistes sólidos, hetereogéneos, de densidad variable y contenido como pasta, que pueden presentar calcificaciones parciales. Constituyen quistes no vitales, envejecidos.

- Tipo V: quistes calcificados. Constituyen, en principio, quistes muertos.

Debería clasificarse como hidatidosis todo caso que, con antecedentes epidemiológicos positivos, es decir, domicilio en áreas endémicas, presente ecografía positiva, o sea, cualquiera de las imágenes ecográficas descritas. Los resultados positivos de pruebas serológicas pueden complementar el diagnóstico de confirmación, pero no son excluyentes (15).

\section{Tamizado de poblaciones}

Se conoce ampliamente la utilidad del tamizado preliminar con fines de detección temprana en poblaciones sin síntomas clínicos. Este método permite mejorar el pronóstico de los pacientes por la ausencia de complicaciones en el momento de la intervención quirúrgica. Por otra parte, aporta datos de vigilancia epidemiológica y facilita la evaluación de programas de control. En el tamizado se usan diversas técnicas serológicas como la prueba
CUADRO 1. Sensibilidad y especificidad de distintas técnicas diagnósticas para la detección de portadores asintomáticos de hidatidosis

\begin{tabular}{lcc}
\hline \multicolumn{1}{c}{ Técnica } & $\begin{array}{c}\text { Sensibilidad } \\
(\%)\end{array}$ & $\begin{array}{c}\text { Especificidad } \\
(\%)\end{array}$ \\
\hline ELISA $a>1$ DE & 84 & 60 \\
ELISA $a>5$ DE & 74 & 84 \\
ELISA a > 8 DE & 63 & 97 \\
Doble difusión 5 & 31 & 100 \\
Ecografía & 100 & 96 \\
\hline
\end{tabular}

ELISA = inmunoadsorción enzimática.

$\mathrm{DE}=$ Desviación estándar.

del látex, HAI y ELISA. Esta última, estandarizada como prueba de referencia, constituye la prueba inmunológica de elección en función de su sensibilidad y especificidad $(3,12,15)$.

En sitios como la Provincia de Río Negro, donde actualmente la prevalencia esperada de resultados positivos en las pruebas ELISA es de 1\%, las reacciones a títulos de más de $8 \mathrm{DE}$ en portadores asintomáticos deben considerarse con precaución. Tienen un valor predictivo positivo de $17 \%$ y deben estudiarse directamente por los métodos usuales de diagnóstico por imagen como la ecografía y la radiografía (y en cuanto sea posible, la tomografía).

En aquellos casos con resultados positivos en el ELISA a títulos entre 5 y 8 DE, el envío a centros de atención terciaria para diagnósticos por imaginología dependerá del criterio epidemiológico del médico actuante, ya que el valor predictivo positivo es de solo 2\% (cuadro 2).

En ambas situaciones, los resultados negativos de la DD5 no permiten descartar la posibilidad de hidatidosis. Con un valor predictivo negativo de $58,9 \%$, se requieren estudios confirmatorios de imaginología. En la detección de portadores asintomáticos cabe poner en duda la validez y la utilidad actuales de la DD5 y otras pruebas serológicas de valor predictivo limitado, como la HAI.

El tamizado ecográfico de portadores asintomáticos permite detectar quistes hidatídicos de tamaño muy re- 
ducido. Ahora es factible diagnosticar a portadores de hidátides de medio a dos centímetros de diámetro.

La capacidad de detección de la ecografía en los tamizados de campo ha resultado $73 \%$ superior a la DD5 en la provincia de Río Negro y $49 \%$ superior al ELISA en Turkana, Kenia. La ecografía tiene una sensibilidad superior a la serología y, por su capacidad para detectar quistes pequeños y producir otra información pertinente, puede usarse con fines de vigilancia epidemiológica y de monitoreo de los programas de control. Sirve, por ejemplo, para evaluar fallas en la interrupción de la transmisión del perro al hombre en zonas sometidas a desparasitación canina regular $(19,21)$. Gracias a la ecografía, el programa de control de Río Negro pudo determinar que la prevalencia de la hidatidosis en portadores asintomáticos de 6 a 14 años de edad se había reducido de $5,6 \%$ en el período de 1984 a 1986 a 1,1\% en el período de 1997 a 1998 y que también se había reducido el tamaño de los quistes detectados. ${ }^{8}$

De acuerdo con esas experiencias, las pruebas de detección por ecografía podrían utilizarse para obtener información básica, como el número de casos de hidatidosis en una región, y para medir la transmisión reciente y evaluar el impacto epidemiológico de los programas de control. Asimismo, en grupos muy expuestos al riesgo de hidatidosis, la ecografía puede emplearse para la detección temprana de la enfermedad. Por ejemplo, en zonas endémicas merece prestarse especial atención al alto porcentaje de casos detectados entre los convivientes de personas operadas por hidatidosis, el cual es estadísticamente superior al de la población general $(12,2 \%$ frente a $4,1 \%$, $P<0,01)$. Debería también evaluarse la situación de las personas que tienen perros portadores de Echinococcus granulosus (20).

\footnotetext{
Frider B, Moguilansky S, Salvitti J, Odriozola M, Cantoni G, Larrieu E. Epidemiological surveillance of human hidatidosis by means of ultrasonography: its contribution to the evolution of control programs. (2000, trabajo no publicado.)
}

CUADRO 2. Valor predictivo (VP) positivo y negativo de los resultados de las distintas técnicas diagnósticas aplicadas para el tamizado de una población no sintomática ajustadas a las prevalencias esperadas en la Provincia de Río Negro, 1980 y 1999

\begin{tabular}{lccccc}
\hline & \multicolumn{2}{c}{$\begin{array}{c}\text { Prevalencia esperada } \\
\text { en 1999: } 1 \%\end{array}$} & & \multicolumn{2}{c}{$\begin{array}{c}\text { Prevalencia esperada } \\
\text { en 1980: } 5 \%\end{array}$} \\
\cline { 2 - 3 } Técnica & VP + & VP - & & VP + & VP - \\
\hline ELISA a > 1 DE & 2,1 & 86,1 & & 9,9 & 5,6 \\
ELISA a > 5 DE & 4,5 & 79,2 & & 19,6 & 78,5 \\
ELISA a > 8 DE & 17,5 & 72,8 & & 52,5 & 71,9 \\
Doble difusión 5 & 100 & 58,9 & & 100 & 57,9 \\
Ecografía & 20,2 & 100 & & 56,8 & 100 \\
\hline
\end{tabular}

El bajo costo operativo del tamizado ecográfico, su alto rendimiento diagnóstico, la rapidez con que se obtienen los resultados y la precisión de estos -el valor predictivo positivo actual en la Provincia de Río Negro es de $20 \%$ y el valor predictivo negativo, de $100 \%$ confirman su viabilidad y utilidad como método de elección en el diagnóstico precoz de la hidatidosis humana en zonas endémicas. Sin embargo, en ambientes rurales donde no se dispone de equipamiento ecográfico para uso sistemático, el ELISA puede utilizarse como prueba de tamizado preliminar si los casos con resultados positivos se envían a centros de mayor complejidad para su estudio con ecografía abdominal y radiografía pulmonar. En la provincia de Río Negro, la población que tuvo resultados positivos en el ELISA fue examinada posteriormente mediante ecografía y la proporción de positivos confirmados fue de $24,5 \%$, cifra superior a la esperable en la población general.
Desde el punto de vista del equipamiento ecográfico, para un eficiente tamizado rural no es necesario contar con instrumental extremadamente complicado. Incluso el personal no especializado puede ser entrenado para lograr buenos resultados usando ecógrafos portátiles sencillos acompañados de pequeños generadores eléctricos, aun en ausencia de instalaciones sanitarias o electrificación urbana (24).

En el cuadro 3 se presenta un resumen de los distintos métodos de tamizado utilizados por los servicios de salud de la provincia de Río Negro en los últimos 20 años.

\section{Tratamiento}

Tradicionalmente, la hidatidosis en el hombre se ha considerado un estado patológico de resolución quirúrgica. La cirugía es todavía el tratamiento de elección según los criterios conservadores en el tratamiento del parásito
CUADRO 3. Resultado de estudios transversales de base y de impacto en una población de 7 a 14 años de edad para evaluar la prevalencia de la hidatidosis humana con diferentes técnicas diagnósticas. Provincia de Río Negro, Argentina

\begin{tabular}{|c|c|c|c|c|c|c|}
\hline \multirow[b]{2}{*}{ Técnica } & \multicolumn{3}{|c|}{ Estudio de base } & \multicolumn{3}{|c|}{ Estudio de impacto } \\
\hline & Año & $\%$ & IC 95\% & Año & $\%$ & IC 95\% \\
\hline Doble difusión 5 & 1980 & 2,1 & $1,9-2,2$ & 1993 & 0,2 & $0,1-0,3$ \\
\hline Ecografía & 1986 & 5,6 & $3,2-9,9$ & 1998 & 1,1 & $0,04-2,7$ \\
\hline ELISA & 1988 & 1,7 & $0,8-3,1$ & 1998 & 1,0 & $0,7-2,2$ \\
\hline
\end{tabular}


CUADRO 4. Resultados preliminares de tratamientos con albendazol en 15 portadores asintomáticos de hidatidosis hepática. Provincia de Río Negro, Argentina, 1997-1998

\begin{tabular}{lc}
\hline $\begin{array}{c}\text { Resultado observado en } \\
\text { los quistes hidatídicos }\end{array}$ & Porcentaje \\
\hline Calcificación de la pared & 5,3 \\
Cambios en el contenido & 21,1 \\
Cambios en las membranas & 26,3 \\
Cambios en las membranas y & \\
$\quad$ el contenido & 10,5 \\
Sin cambios & 31,6 \\
\hline
\end{tabular}

$(25,26)$. La búsqueda de casos asintomáticos mediante el tamizado ecográfico o serológico ha tenido como objeto la pronta intervención quirúrgica para disminuir el riesgo del paciente, el tiempo de internación y las posibles complicaciones (3).

En los últimos años han comenzado a aplicarse con éxito la quimioterapia con mebendazol y albendazol y los tratamientos quirúrgicos poco invasores como la punción-aspiración-inyecciónreaspiración (PAIR) en pacientes con síntomas de la enfermedad. Se han registrado cambios indicativos de pérdida de vitalidad del quiste en las imágenes ecográficas de 54 a $71 \%$ de los pacientes tratados con albendazol y se han publicado algunos trabajos sobre las posibilidades de la PAIR en el tratamiento de la hidatidosis humana sintomática (27-31).

Hay mucho menos información sobre el tratamiento de portadores asintomáticos. Sin embargo, en la provincia de Río Negro se cuenta con información preliminar que indica $69 \%$ de respuestas favorables al tratamiento con albendazol en 15 portadores detectados en tamizados de campo (cuadro 4) (29). Diez y ocho meses después de haberse aplicado la técnica de PAIR, se observó que el volumen medio de los quistes se había reducido $66 \%$ en 38 pacientes asintomáticos portadores de 60 quistes $(63 \%$ vitales y $37 \%$ no vitales). También se observaron cambios favorables en las imágenes de ecografía y tomografía. El promedio de días de internación de los pacientes tratados con PAIR fluctuó entre 0 y 4 (32).

Puesto que no hay ningún tratamiento que asegure una respuesta similar en todos los portadores de hidatidosis, en la elección del tratamiento de un caso asintomático es necesario considerar los aspectos siguientes: a) la vitalidad del parásito, b) el tratamiento del parásito, c) las posibilidades de siembra quirúrgica, d) la resolución de la cavidad, e) la mortalidad y la morbilidad, f) el costo del tratamiento y g) la reinserción social del paciente.

En función de ello, los servicios hospitalarios de la provincia de Río Negro definieron un protocolo operativo ba- sado en el diagnóstico precoz mediante estudios ecográficos de toda la población escolar de zonas de riesgo. El tratamiento de los casos detectados se basa en el siguiente algoritmo (figura 1):

- Tipo Ia: seguimiento ecográfico (baja proporción de complicaciones).

- Tipos Ib a III: tratamientos no convencionales de quimioterapia con albendazol y, en caso de respuesta negativa, PAIR. De no remitir con este último tratamiento, se aplica cirugía convencional (quistes con capacidad potencial de complicación).

- Tipo IV: seguimiento y control, sin tratamiento (quistes de poca vitalidad).

FIGURA 1. Protocolo para el monitoreo y el tratamiento de quistes hidatídicos en pacientes asintomáticos de los servicios hospitalarios de Río Negro, Argentina

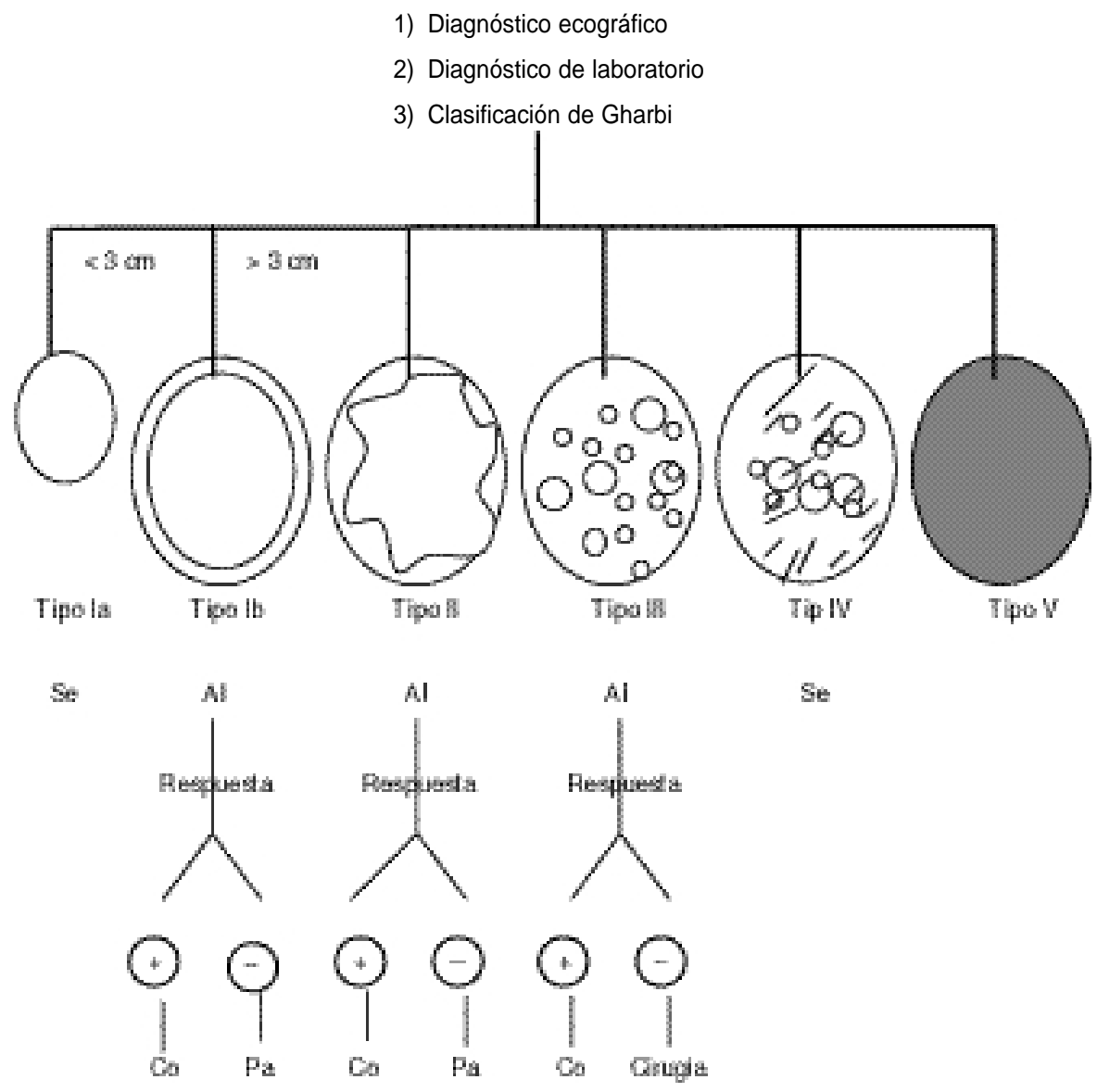

$\mathrm{Se}=$ seguimiento ecográfico.

$\mathrm{Al}=$ tratamiento con albendazol.

$\mathrm{Co}=$ control.

$\mathrm{Pa}=$ punción-aspiración-inyección-reaspiración. 
- Tipo V: no requieren servicios de control regular, sin tratamiento (quistes muertos).

Los criterios de seguimiento se basan en el control ecográfico para verificar las modificaciones de tamaño y el estado de membranas y contenido (modificaciones cualitativas y cuantitativas) y seguimiento serológico.

\section{Conclusión}

Desde el punto de vista de la atención a las personas, la búsqueda activa y sistemática de portadores humanos asintomáticos es de reconocida importancia para definir el perfil epidemiológico de la enfermedad en una zona endémica. $(33,34)$. Esa búsqueda también está dirigida a promover la cirugía temprana, que tiene mejor pronóstico quirúrgico por la ausencia de complicaciones. Con esta estrategia se han logrado disminuciones notables en los tiempos de internación y en las tasas de letalidad (3).

Los conocimientos actuales, sin embargo, revelan que un alto porcentaje de portadores asintomáticos de quistes pequeños no sufren durante su vida las consecuencias de la enfermedad (7).

La posibilidad del tratamiento con medicamentos y de intervenciones quirúrgicas poco invasoras, incluso en combinación (35), se suman actualmente al desarrollo y la accesibilidad de las técnicas de imaginología para

\section{REFERENCIAS}

1. Ivanisevich O, Rivas C. Equinococosis hidatídica, Tomo I. Buenos Aires: Ministerio de Educación y Justicia de la República Argentina:1961.

2. Frider B, Larrieu E, Corti O. Frecuencia de las localizaciones hepática y pulmonar del quiste hidatídico en pacientes sintomáticos y en portadores asintomáticos de áreas endémicas. Rev Iber Parasitol 1988;48:149-153.

3. Larrieu E, Guarnera E, Costa M, Álvarez J, Cantoni G, Pérez A, Giménez N. Control de la hidatidosis en la Provincia de Río Negro. Evaluación actividades atención médica. Rev Sanid Hig Publ 1993;5:377-384.

4. Mlika N, Larouze B, Gaudebout C, Braham B, Allegue M, Dazza M, et al. Echotomographic and serologic screening for hydatidosis in a Tunisian Village. Am J Trop Med Hyg 1986; 35:815-817.

5. Perdomo R, Parada R, Álvarez C, Cattivelli D, Geninazzi H, Ferreira C, et al. Estudio epidemiológico de hidatidosis: detección precoz por ultrasonido. Rev Med Uruguay 1990; 6: 34-47.

6. Frider B, Losada C, Larrieu E, Zavaleta O. Asymptomatic abdominal hydatidosis detected by ultrasonography. Acta Radiol 1988; 29:431-434.

7. Frider B, Larrieu E, Odriozola M. Long term outcome of asymptomatic liver hydatidosis. J Hepatol 1999;30:228-231

8. Larrieu E. Bases biológicas y epidemiológicas para el control de la hidatidosis/equinococosis. Veterinaria Argentina 1991;54:612-621.

9. Coltorti E, Varela Díaz V. Detection of antibodies against Echinococcus granulosus arc 5 antigens by double diffusion test. Trans R Soc Trop Med Hyg 1978;72:226-229.

10. Zamorano C, Contreras M, Sánchez A, Bahamondes M, Sandoval L. Estudio seroepide- miólogico de la hidatidosis y triquinosis humana mediante la reacción de hemaglutinación indirecta en la comuna de San Juan de la Costa, Chile, 1990-1991. Bol Chil Parasitol 1991;46:82-84.

11. Coltorti E, Fernández E, Guarnera E, Lago J, Iriarte J. Field evaluation of an enzyme immunoassay for detection of asymptomatic patients in a hydatid control program. Am J Trop Med Hyg 1988;38:603-607.

12. Coltorti E. Standardization and evaluation of an enzyme immunoassay as a screening test for the seroepidemiology of human hydatid. Am J Trop Med Hyg 1986;35:1000-1005.

13. Shambesh M, Craig P, Gusbi A, Wen H. Immunoblot evaluation of the 100 and $130 \mathrm{Kda}$ antigens in camel hydatid cyst fluid for the serodiagnosis of human cystic echinococcosis in Lybia. Trans R Soc Trop Med Hyg 1995;89: 276-279.

14. Arienti H, Guignard S, Rinakldi D, Elbarcha O. Comparison of two serologic methods for the diagnosis of hydatidosis. Rev Panam Salud Publica 1997;5:376-379. Bianchi C, Moguilansky A. Evaluación de ELISA y DD5 en el diagnóstico de la hidatidosis humana en población asintomática. Rev San Hig Publ 1994;68:393-398.

16. Thompson R, Lymbery A. Equinococcus and hydatid disease. London: Ed Cab International; 1995.

17. Gharbi H, Hassine W, Brauner M, Dupuch K. Ultrasound examination of the hydatic liver. Radiology 1981;139(2):459-463.

18. Frider B, Ledesma C, Odriozzola M, Larrieu tico precoz de la hidatidosis humana. Acta Gastroenterol Lat Amer 1992;20:13-15.
15. Larrieu E, Dapcich C, Guarnera E, Coltorti E, E. Especificidad de la ecografía en el diagnós- establecer esquemas de búsqueda activa e iniciar el seguimiento longitudinal para determinar la evolución del quiste y la instauración de tratamientos no invasores, todo a un costo aceptable para los servicios de salud.

Agradecimiento. Los autores dan las gracias a los doctores Víctor Varela Díaz y Emilio Coltorti de la Organización Panamericana de la Salud y al doctor Peter Schantz de los Centros para el Control y la Prevención de Enfermedades (EUA), por el asesoramiento e impulso que brindaron a los equipos profesionales de la Provincia de Río Negro en el desarrollo de estrategias de diagnóstico precoz y tratamiento oportuno de las personas.
19. Frider B, Larrieu E, Aguero A, Catastro ecográfico de hidatidosis en un área endémica, estudio comparativo con DD5. Rev Iber Parasitol 1986;46:257-266.

20. Larrieu E, Frider B, Andreani G, Andreani G Aquino A, De La Fuente R. Hidatidosis humana: ecografía de campo para la determinación de grupos de alto riesgo en la evaluación de un programa de control. Rev Inst Trop Sao Paulo 1989;31:267-270.

21. Macpherson C, Romig T, Zeyhle E, Rees P, Werw J. Portable ultrasound scanner versus serology in screening for hydatid cyst in a nomadic population. Lancet 1987;259-261.

22. Saint Martin G, Chiessa J. Falling snowflakes, an ultrasound sign of hydatid sand. J Ultrasound Med 1984;3:256-257.

23. Del Carpio M, Moguilansky S, Costa M, Panomarenko H, Bianchi C, Bendersky S, et al. Diagnosis of human hydatidosis: predictive value of the rural ultrasonographic survey in an apparently healthy population. Medicina 2000. En prensa.

24. Saint Martin G, Larrieu E, Chiessa C, Dapcich C. Ultrasound scanning as a screening technique for hydatidosis in developed countries. J Clin Ultrasound 1988;16:233-237.

25. Mercapide C, Giménez R, Pereyra R, Pérez C, Michelena F. Tratamiento de la hidatidosis hepática. Prensa Med Argent 1994;81:275-281.

26. Vagianos C, Karavias D, Kakkos S, Vagenas $\mathrm{C}$, Andoulakis J. Conservative surgery in the treatment of hepatic hydatidosis. Eur J Surg 1995;161:415-420.

27. Gil Grande L, Rodríguez Caabeiro F, Prieto J, Sánchez Ruano J, Brasa C, Aguilar L, et al. Randomised controlled trial of efficacy of albendazol in intra-abdominal hydatid disease. Lancet 1993;342:1269-1272. 
28. Horton R. Albendazole in treatment of human cystic echinococcosis: 12 years of experience. Acta Trop1997;64:79-93.

29. Salvitti J, Mercapide C, Pereyra R, Odriozola M, Del Carpio M, Larrieu E. Treatment with albendazole of asymptomatic hydatic carriers. Arch Inter Hid 1999:33:205-206

30. Filice C, Brunetti E. Use of PAIR in human cystic echinococcosis. Acta Trop 1997;64: 95-107.

31. WHO informal working group on echinococcosis: guidelines for treatment of cystic and alveolar echinococcosis in humans. Bull World Health Org 1996;74:231-242.
32. Pelaez V, Kugler C, Del Carpio M. Liver hydatid disease: results of percutaneous treatment. Bol Chil Parasitol 1999;54:63-69

33. Selentis J, Karpathios T, Fretzayas A, Nicolaidou P, Matsaniotis N. Hydatid disease in asymptomatic young carriers in northern Greece. Am J Trop Med Hyg 1983;32:14621463.

34. Schantz P, Williams J, Posse R. Epidemiology of hydatid disease in southern Argentina. Am J Trop Med Hyg 1973;22:629-641.

35. Khuroo M, Dar M, Yatoo S, Zagar S, Javaid G, Khan A, et al. Percutaneous drainage versus albendazole therapy in hepatic hydatidosis: a prospective, randomized study. Gastroenterology 1993;104:1452-1459.

Manuscrito recibido el 18 de mayo de 1999 y aceptado para publicación, tras revisión, el 8 de junio de 2000.

ABSTRACT Until a short time ago, hydatidosis was considered a pathology that could only be resolved surgically. However, in recent years progress has been made with the epidemiology, diagnosis, and treatment of the disease, and new information on the nat-

Asymptomatic carriers of hydatidosis: epidemiology, diagnosis, and treatment ural history of hydatidosis has helped define new criteria for its treatment. It is now known that as many as $67 \%$ of the carriers of liver cysts who are asymptomatic remain so throughout their lives. This situation produces special results in immunologic testing. Enzyme-linked immunosorbent assay (ELISA) has a sensitivity of $63 \%$ and a specificity of $97 \%$ with asymptomatic carriers, while the double diffusion arc 5 test (DD5) achieves a sensitivity of only $31 \%$ with the same population. On the other hand, imaging studies based on ultrasonography have become the method of choice to detect asymptomatic carriers. Ultrasonography studies are $49 \%$ to $73 \%$ more sensitive than serological tests, and they can even be used as a part of epidemiological surveillance systems and to monitor control programs. Treatment schemes have also been modernized. Treating asymptomatic carriers chemotherapeutically with albendazole produces favorable results in as many as $69 \%$ of cases, while such minimally invasive surgical treatments as puncture-aspiration-injection-reaspiration (PAIR) reduce average cyst volume by as much as $66 \%$. These factors have made it possible for hospital services in the province of Río Negro, Argentina, to establish a treatment scheme for asymptomatic carriers. It is based on the monitoring of small cysts (type Ia on the modified Gharbi scale); initial treatment with albendazole, followed by PAIR if there is no response, in larger or more complex cysts (types Ib, II, and III); and follow-up of inviable or dead cysts (types IV and V). 